\title{
Host cell changes induced during Severe Acute Respiratory Syndrome (SARS) coronavirus infection.
}

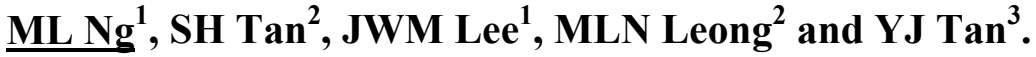 \\ ${ }^{1}$ Flavivirology Laboratory, Department of Microbiology, 5 Science Drive 2, National \\ University of Singapore, Singapore 117597. ${ }^{2}$ Electron Microscopy Unit, 12 Medical \\ Drive, Faculty of Medicine, National University of Singapore, Singapore 117597. \\ ${ }^{3}$ Institute of Molecular and Cell Biology, 61 Biopolis Drive, Proteos, Singapore 138673.
}

\section{Introduction}

A new virus infection started in southern China in November 2002 and subsequently spread to Hong Kong, Vietnam, Canada and Singapore. The Severe Acute Respiratory Syndrome (SARS) virus was identified to be a new virus that belongs to the coronavirus family (Drosten et al., 2003; Ksiazek et al., 2003). Unlike the other members which cause mild respiratory symptoms (Holmes, 1990), the SARS coronavirus result in fatalities ranging from 10 to $15 \%$ of the infected patients depending on the age group. The replication cycle was similar to the other coronaviruses in that the latent period was about 6 hours in cell cultures however the production of progeny virus was very efficient (Sturman \& Takemoto, 1972; Ng et al., 2003). Microscopy investigation was undertaken to see if any observed host changes induced by the virus infection could help explain the severity of the disease.

\section{Methods and Materials}

The TEM, AFM and SEM were used to study the morphological changes in the infected cells. Immuno-TEM was also used to localize a unique SARS virus protein $3 a$ (U274) in relation to the induced morphological changes. Attempts were made to look for virus localization and induced morphological changes in the autopsy lung tissues using the TEM.

\section{Results and conclusions}

In in vitro studies the SARS virus was found to replicate at great speed and produced high numbers of progeny virus particles within 12 to 15 hours post infection. The early changes entail the appearance of the large vacuoles enclosing large myelin-like membrane whorls. These membranes could have a role in the early event of replication as they are seen closely associated with the uncoated virus nucleocapsids. These membrane structures remained prominent during the first 10 hours of infection and diminished in quantity after the nucleocapsids were assembled in the vicinity of the swollen Golgi complexes. After 12 hours of infection, numerous newly formed nucleocapsids were observed maturing at the Golgi complexes. These mature virus particles were transported to the exterior by vacuoles walls fusing with the plasma membrane. 
Transmission electron microscopy was also conducted on some autopsy lung tissues from Singapore fatalities. There was no prevalent presence of SARS virus in these tissues. One of the reasons could be that the tissues were not in good condition when received for TEM processing. Nevertheless some virus particles were observed together with copious amount of collagens in these tissues.

The quick replication process generating large numbers of progeny virus is not common for the other members of the coronaviruses. Tan and colleagues (2004) discovered a novel protein U274 (now referred to as 3a) in this virus. The possibility that this protein could have an essential role in the replication/assembly of the virus was investigated. Transfected cells were examined under the TEM for morphological changes resulting from the expression of this protein. The expressed protein form crystalline array complexes at the perinuclear region. The protein was identified using immuno-TEM. The general morphology of the transfected cells was dramatically changed. The cells became rounded with highly vacuolated cytoplasm. From the preliminary screening of these cells it could be deduced that this novel protein may be one of the virus products that induces cytopathic effects in the cells.

\section{Acknowledgement}

This work was supported by a grant (R-182-000-055-112) from the National University of Singapore. We are grateful to Dr Ling Ai Ee, Singapore General Hospital and Dr Angela Chong, Tan Tock Seng Hospital for providing the lung tissues.

\section{References}

1. Drosten C, Gunther S, Preiser W, van der Werf S, Brodt HR, Becker S, et al. Identification of a novel coronavirus in patients with severe acute respiratory syndrome. N Engl J Med (2003) 348:1967-1976.

2. Ksiazek TG, Erdman D, Goldsmith CS, Zaki SR, Peret T, Emery S, et al. A novel coronavirus associated with severe acute respiratory syndrome. N Engl J Med (2003) 348:1953-1966.

3. Holmes KV. Coronaviridae and their replication. In Virology, $2^{\text {nd }}$ edition, edited by BN Fields, DM Knipe, PM Howley, RM Channock, TP Monath, JL Melnick, B Roizman and SE Strauss (1990) pp. 841-856, New York: Raven Press.

4. Sturman LS, Takemoto KK. Enhanced growth of a murine coronavirus in transformed mouse cells. Infect Immun (1972) 6:501-507.

5. Ng ML, Tan SH, See EE, Ooi EE, Ling AE. Prolific replication of SARS coronavirus in E6 cells. J Gen Virol (2003) 84:3291-3303.

6. Tan YJ, Teng E, Shen S, Tan THP, Goh PY, Fielding BC, et al. A novel SARS coronavirus protein $\mathrm{U} 274$, is transported to the cell surface and indergoes endocytosis. J Virol (2004) 78: 6723-6734. 